Costume et déguisement dans le théâtre de Shakespeare et de ses contemporains

\title{
« Armed at point » et « night gown » : les oripeaux du spectre et l'exception Hamlet
}

\section{Pierre Kapitaniak}

\section{(2) OpenEdition Journals}

Édition électronique

URL : http://journals.openedition.org/shakespeare/1456

DOI : $10.4000 /$ shakespeare. 1456

ISSN : 2271-6424

Éditeur

Société Française Shakespeare

Édition imprimée

Date de publication : 20 décembre 2008

Pagination : 35-47

ISBN : 2-9521475-5-8

\section{Référence électronique}

Pierre Kapitaniak, « «Armed at point » et « night gown » : les oripeaux du spectre et l'exception Hamlet ", Actes des congrès de la Société française Shakespeare [En ligne], 26 | 2008, mis en ligne le 20 décembre 2008, consulté le 21 avril 2019. URL : http://journals.openedition.org/shakespeare/1456 ; DOI : 10.4000/shakespeare.1456 


\section{Costume et déguisement dans le théâtre de Shakespeare et de ses contemporains}

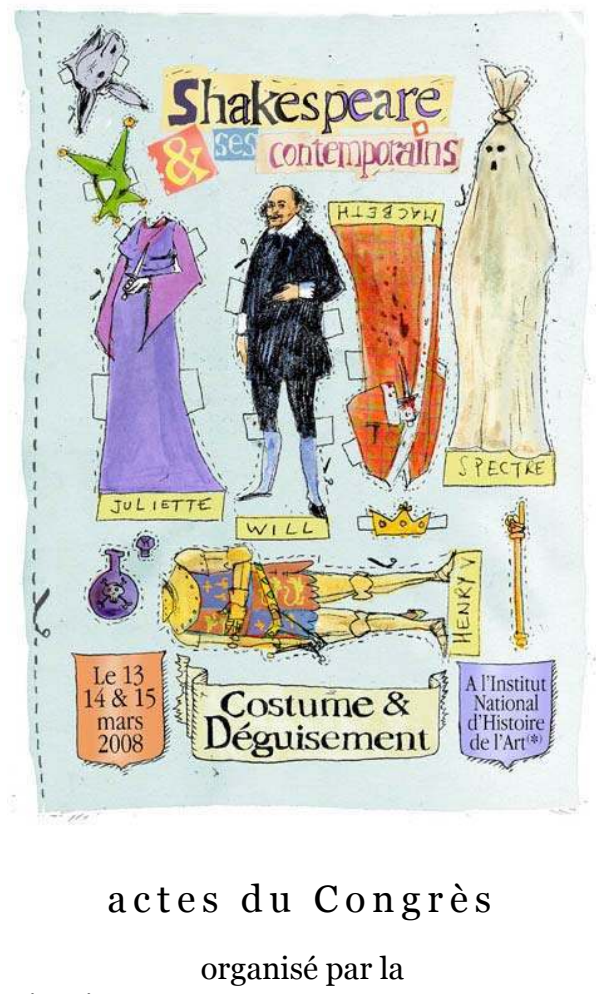

SOCIÉTÉ FRANÇAISE SHAKESPEARE

les 13,14 et 15 mars 2008

textes réunis par

Pierre KAPITANIAK

sous la direction de

Jean-Michel DÉPRATS 
COUVERTURE :

Edouard Lekston,

William en carton et ses habits à découper, 2008 edlek@free.fr

conception graphique et logo

Pierre Kapitaniak

\section{(C) 2008 Société Française Shakespeare}

Institut du Monde Anglophone

Université de Paris III - Sorbonne Nouvelle

5 rue de l'École de Médecine

75006 Paris

www.societefrancaiseshakespeare.org

Tous droits de traduction, de reproduction et d'adaptation réservés pour tous les pays 


\title{
« ARMED AT POINT » ET « NIGHT GOWN 》 : LES ORIPEAU X DU SPECTRE ET L'EXCEPTION HAMLET
}

\author{
Pierre KAPITANIAK
}

Les apparitions du spectre dans Hamlet - tantôt en armure, tantôt en vêtement d'intérieur - ont tellement marqué les esprits, tant à l'époque que de nos jours, qu'on oublie souvent à quel point celui-ci s'éloigne des façons habituelles de représenter un revenant sur les scènes élisabéthaines. En puisant dans le contexte de l'époque et notamment dans les divers écrits sur les apparitions et la démonologie, la présente communication tentera de déterminer les costumes propres aux spectres avant et après Hamlet pour faire apparaître l'originalité de Shakespeare ainsi que son l'influence sur la tradition spectrale et sur les dramaturges jacobéens et caroléens.

The apparitions of the ghost in Hamlet - first in armour, then in a night gown - have left such a mark on the audiences' minds, both at the time and nowadays, that one easily tends to forget how far this ghost is from the usual ways of representing a revenant on Elizabethan stages. Using the demonological context of the period, the present paper will try to determine the typical ghost costumes before and after Hamlet to emphasise Shakespeare's originality as well as his influence on Jacobean and Carolean playwrights.

orsqu'on songe au spectre du vieux roi dans Hamlet, on se figure en général une sombre silhouette en armure, arpentant les murailles d'Elseneur, dans des volutes de brume, hérauts de l'aube imminente. Mais ce cliché nous vient de la tradition romantique. Rien de tel sur la scène du Globe au début du XvII ${ }^{\mathrm{e}}$ siècle. Pas de murailles. Pas de brume. Probablement une armure de pacotille. Mais surtout en ce tout début du siècle - et du Globe - l'apparition en armes fait sensation. On n'avait encore jamais vu cela ! Voici un revenant qui revêt une noble form g guerrière («fair and warlike form » I.i.45) dans ses premières visites. Celle-ci n'est pas le fruit d'une didascalie, mais nous parvient par le commentaire de Horatio après le départ du spectre :

Such was the very armour he had on

When he th'ambitious Norway combated.

(I.i.59-60)

Elle est ensuite longuement développée lorsque Horatio, secondé par les deux gardes, rend compte à Hamlet de ce qu'ils ont vu, en soulignant les détails comme armé de pied en cape, armé de haut jusqu'en bas, de la tête aux pieds, ou encore sa visière levée :

Hor. A figure like your father, Armed at point, exactly, cap-à-pie, Appears before them [...] 


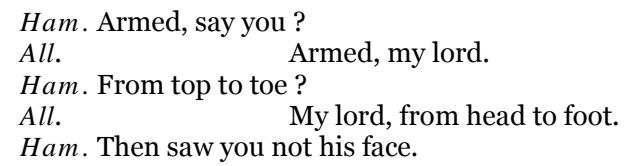

not his face.

Hor. O yes, my Lord, he wore his beaver up.

Hamlet ne peut s'empêcher d'interpréter cette apparition extraordinaire comme un mauvais présage («My father's spirit in arms ! All is not well », I.ii.254) et lorsqu'il a enfin l'occasion de s'adresser lui-même au fantôme de son père, il ne manque pas de relever une dernière fois l'armure complète qui semble directement motiver les questions et les doutes qui l'assaillent :

What may this mean,

That thou, dead corpse, again in complete steel,

Revisits thus the glimses of the moon...

(I.iv.32-34)

Après des apparitions en armure tout au long du premier acte, le fantôme qui apparaît dans la chambre de la reine est décrit par son fils en ces termes : "My father in his habit as he lived » (III.iv.126). La didascalie du premier in-quarto de 1603 nous apprend qu'il s'agit de sa robe de nuit («the ghost in his night gowne»), ce qui signifie que ce spectre-là ne porte pas un déguisement spécifique, et que ce n'est pas dans les vêtements qu'il faut chercher les signes de sa condition de trépassé.

On peut se demander si l'idée d'insister sur la description du spectre ne vient pas justement du fait qu'il s'agit d'une chose inhabituelle dans la tradition théâtrale de l'époque. La surenchère des descriptions et des réactions, qui sont autant de tentatives d'interprétation de cet accoutrement, ferait ainsi partie intégrante de l'étonnement: les personnages sont confrontés à une apparition surnaturelle, mais au-delà du caractère exceptionnel de ce statut, le dramaturge offre au public un commentaire sur l'originalité de son procédé.

En effet, si l'on interroge l'ensemble du corpus théâtral de l'époque, Hamlet est la première pièce à mentionner un spectre en armure... et une des seules. Ce costume semble avoir été cher à Shakespeare, qui le reprend quelques années plus tard dans Cymbeline (1609), où il fait apparaître le père de Posthumus en guerrier (« attired like a warrior », v.iii.123 ${ }^{\mathrm{D}}$ ). 
À part Shakespeare, seules deux pièces caroléennes mettent en scène des spectres semblables et toutes deux affichent ouvertement une dette fort lourde à leur modèle.

La première, Fuimus Troes (1625) de Jasper Fisher ${ }^{1}$, montre dans le prologue des spectres qui apparaissent aussi armés que le vieux Hamlet : « in compleate armour, and with swords drawne » (Prol. $\left.\mathrm{o}^{\mathrm{D}}\right)$, puis plus tard au cours de la pièce, les mêmes spectres réapparaissent dans la chambre d'un vivant qui, lui, est en robe de nuit, comme pour rappeler sa source d'inspiration.

Un écho comique du changement de costume qui opère dans Hamlet se trouve dans The Fatal Contract (1639) de William Heminges $^{2}$. Un premier personnage s'y déguise en fantôme en revêtant une armure, mentionné deux fois par les témoins («warlike form », « in armes », III.i.3o \& 40), ce que confirme aussitôt la didascalie (« as in the Princes Armour » III.i.47 ${ }^{\mathrm{D}}$ ). À l'acte suivant, un autre personnage se fait passer pour le spectre de son père en enfilant les vêtements du défunt, ce que précisent deux didascalies («his Fathers Gown and Robes », IV.iii. $93^{\mathrm{D}}$, « he puts off his robes » IV.iii.105 ${ }^{\mathrm{D}}$ ).

Un tel choix prouve donc la dette à Shakespeare mais ne correspond pas nécessairement à la pratique générale. Alors en quoi consiste-t-elle? Faute d'ouvrages théoriques contemporains sur les pratiques théâtrales en Angleterre, il faut rechercher des traces de mises en scène dans les pièces elles-mêmes (didascalies et références textuelles), ainsi que dans d'autres documents tels les inventaires des théâtres, les recueils d'histoires prodigieuses ou encore les traités démonologiques, et bien entendu l'iconographie.

\section{Être ou ne pas être $n u$}

Même si c'est le vieux roi dans Hamlet qui est passé à la postérité comme la quintessence du spectre élisabéthain, le seul témoin d'éventuelles pratiques théâtrales est un autre spectre, oublié aujourd'hui, et qui doit pourtant beaucoup à son illustre prédécesseur. En effet, en matière de revenants, le théâtre n'en fournit qu'une seule

\footnotetext{
${ }^{1}$ Jasper Fisher, Fuim us Troes or the True Trojans, Londres, I. L. pour Robert Allot, 1633.

${ }^{2}$ William Heminges, The Fatal Contract, A French Tragedy, Londres, J. M., 1653.
} 
image, figurant sur la vignette qui illustre la préface d'une tragédie de William Sampson, The Vow Breaker (1625) ${ }^{3}$ (Ill.1).

Le tableau est divisé en quatre parties correspondant aux moments clefs de la tragédie. En haut à gauche est représenté le jeune Bateman juste après son suicide. Son corps est dénudé, et Bateman porte encore au cou la corde avec laquelle il s'est pendu. À son poignet gauche, il porte le bracelet représentant sans doute la pièce d'or qu'il avait offerte à Anne en gage de son amour. De l'autre main, il pointe vers ce bijou en prononçant la phrase qui revient tout au long de la pièce : " Thinke on thy promise alive or dead I must and will enjoy thee $»$.

La convention de représenter l'âme par un petit personnage nu remonte au Moyen Âge ${ }^{4}$, mais on retrouve ce type de figuration dans d'autres gravures de la première moitié du XVII siècle. Le plus ancien est Ratseis Ghost $(1605)^{5}$, un court texte qui relate les aventures d'un fameux voleur exécuté cette même année (IIl.2). Une représentation similaire orne le poème que Richard Niccols dédie à la mémoire du poète Thomas Overburie, tombé en disgrâce, puis victime d'une intrigue qui conduisit à son empoisonnement en 1613. Dans Sir Thomas Overburies Vision (1616) (Ill.3), la gravure qui figure en frontispice, est également reprise dans le corps du texte, à l'endroit exact où le poème décrit l'apparition d'Overburie :

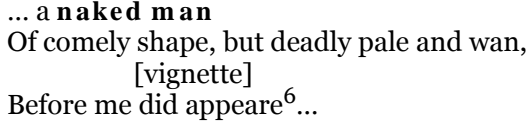

Même si l'image ne semble pas avoir été conçue spécialement pour ce texte, une note marginale placée à côté de la vignette à la page 4 ( « Sir Thom as Overburies ghoast ») précise qu'elle illustre ce spectre-là, et de plus le texte mentionne la nudité de l'apparition. Enfin, on peut

\footnotetext{
3 William Sampson, The Vow Breaker. Or, The Faire Maide of Clifton, Londres, John Norton pour Roger Ball, 1636.

4 Voir Danièle Alexandre-Bidon (éd.), À réveiller les morts, illustrations entre p. 160 et 161; Jean-Claude Schmitt, Les revenants, illustrations entre p. 118 et 119; Claude Lecouteux, Chasses fantastiques et cohortes de la nuit au Moyen Âge, p. 208 ; Daniel Fabre (éd.), Le retour des morts, illustrations entre p. 48 et 49.

5 Ratseis Ghost, or The second Part of his madde Prankes and Robberies (1605)

${ }^{6}$ Sir Thomas Overburies Vision; With the ghoasts of Weston, M. Turner, the late Lieftenant of the Tower, and Franklin [Oxford], P. M \& T. I., 1616, p. 4-5.
} 
Ill.1: William Sampson, The Vow Breaker, Londres, John Norton, 1636, p. 3.

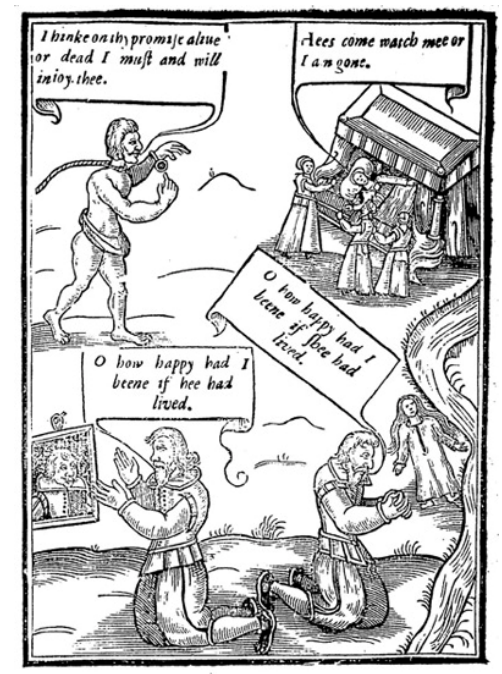

Ill.2: Ratseis Ghost, or The second Part of his madde Prankes and Robberies, Londres, V[alentine] S[immes] pour John Hodges, 1605.

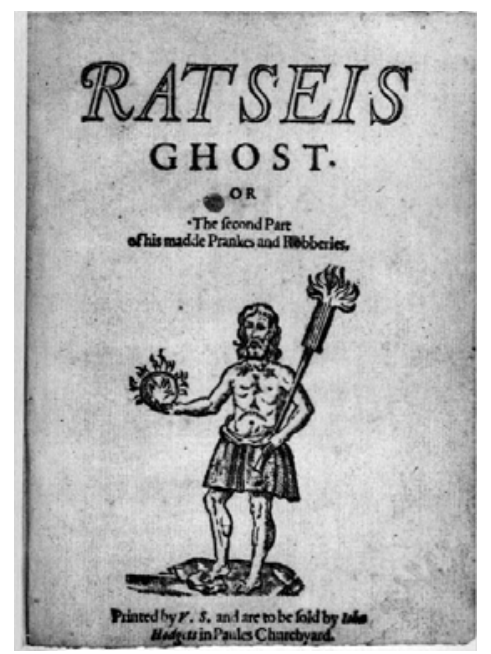


IIl.3: Richard Niccols, Sir Thomas Overburies Vision, [Oxford], P. M \& T. I., 1616

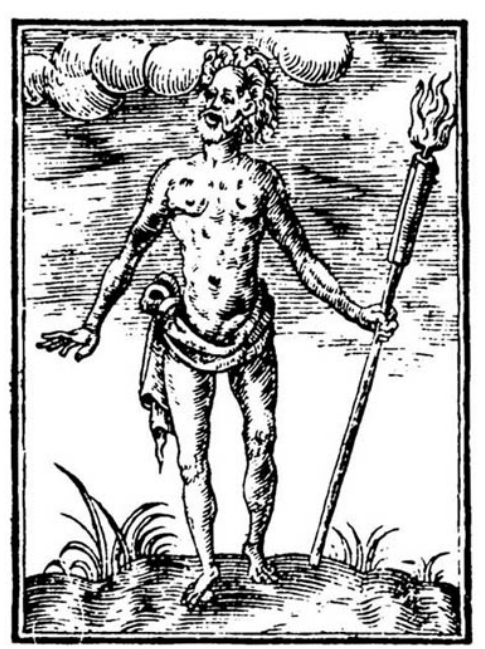

Ill.4: Canterburies amazement: or The Ghost of the yong fellow Thomas Bensted, [Londres], F. Coules, 1641.

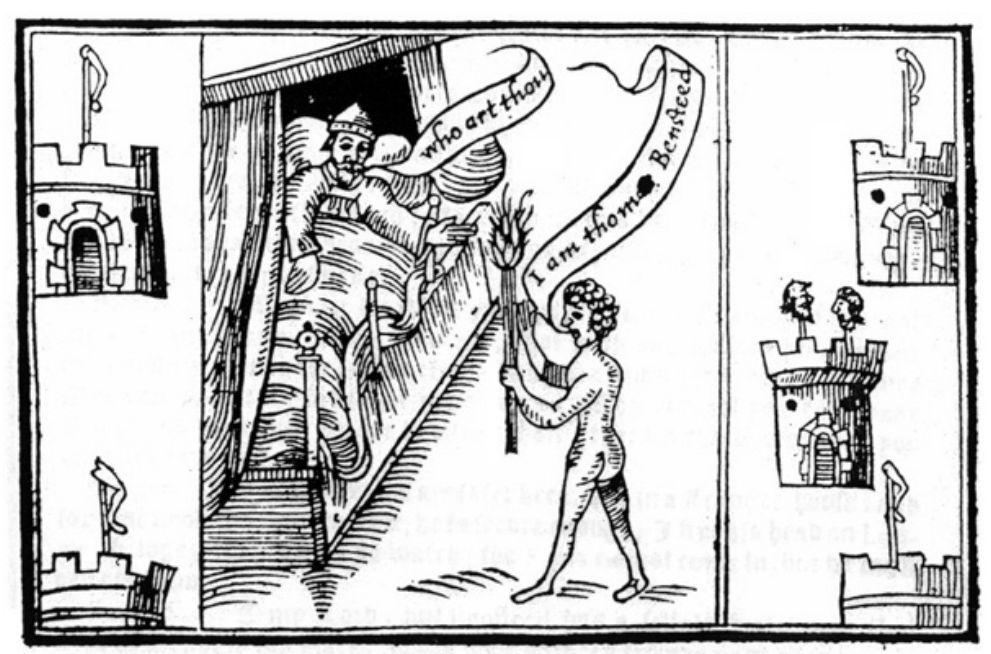


Ill.5: Pierre Boaistuau, Histoires prodigieuses, Paris, Vincent Sertenas, 1560, p. 105 .

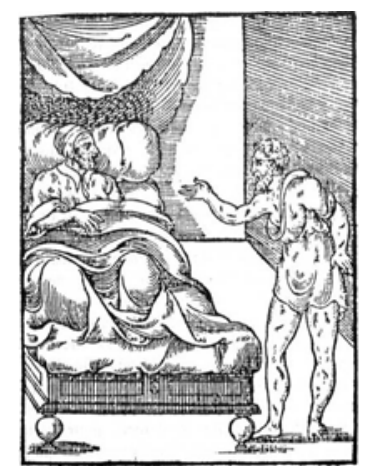

Ill.6: François de Belleforest, Histoires prodigieuses, Paris, Veuve de Gabriel Buon, 1598, p. 588 .

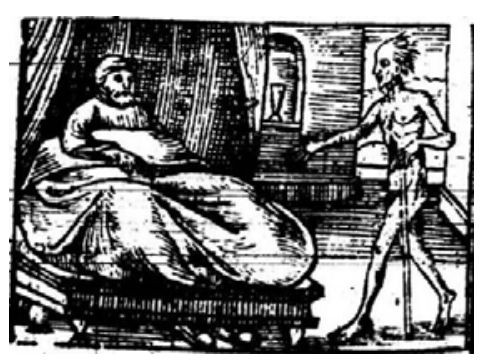

Ill.7: [T] he [de]puties ghost : or [his ?] apparition to the [l]ord of Canterbury in the Tower, 1641 , broadsheet

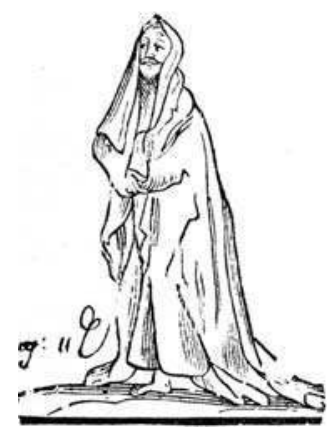


Ill.8: The Earle of Straffords ghost, Complaining of the Cruelties of his Countrey-men, Londres, G. Bishop, August 221644.

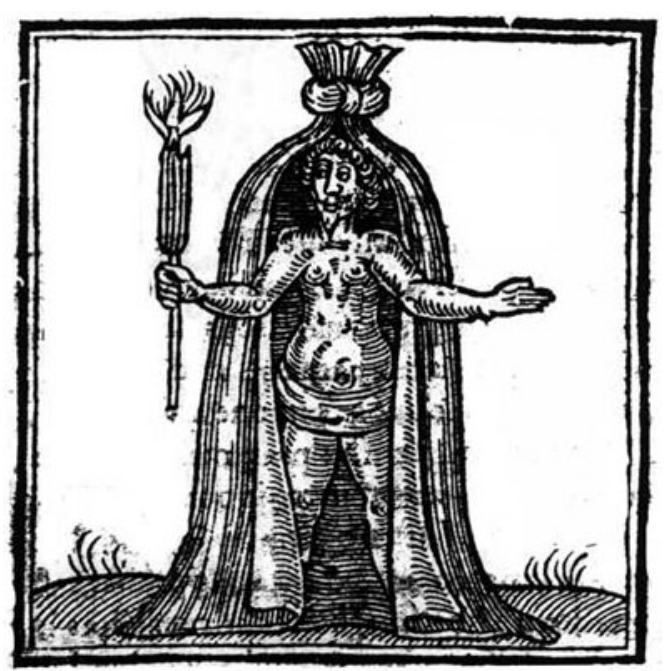

Ill.9: Strange Apparitions, or The Ghost of King James, Londres, J. Aston, 1642 .

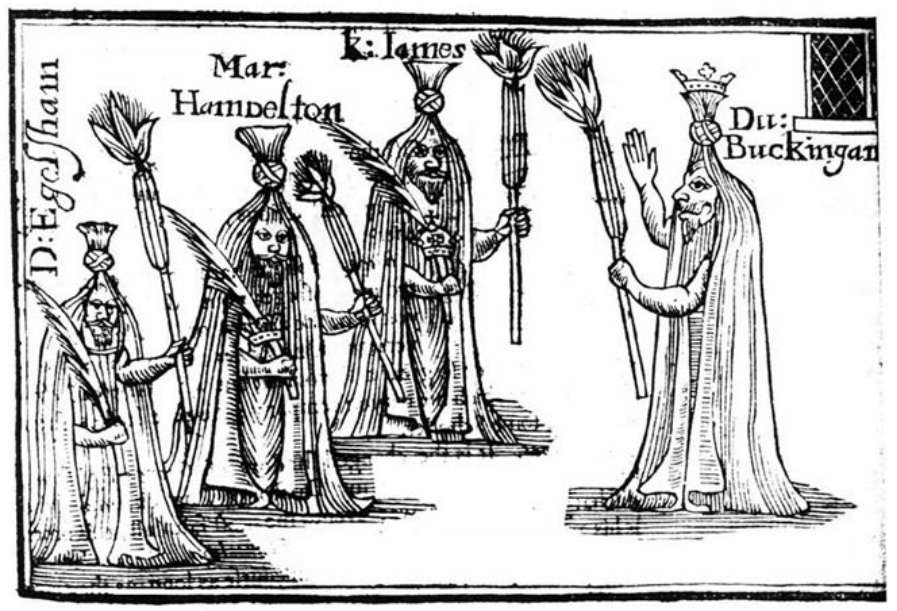


mentionner également un pamphlet plus tardif, datant de 1641, où la vignette montre un autre revenant tout nu, celui de Thomas Bensted fraîchement exécuté. (Ill.4)

On peut se demander si ce type de représentation ne fut pas influencé par une des gravures originales qui accompagnaient le recueil d'Histoires prodigieuses de Pierre Boaistuau. Publié à Paris en 1560, il fut traduit en anglais par Edward Fenton sous le titre Certaine secrete wonders of Nature, containing a description of sundry strange things, seming monstrous, et publié en 1569 par Henry Bynneman7. Le spectre qui figure sur la première page du chapitre consacré aux apparitions porte un vêtement un peu plus fourni que les autres, mais qui laisse tout de même une grande partie de son corps dénudé (Ill.5). Cette gravure fut reprise et refaite dans les éditions augmentées par Tesserand puis par Belleforest par la suite avec des variantes significatives, laissant place dans une des versions à un corps entièrement nu (Ill.6).

Même si la nudité n'est pas une impossibilité sur les scènes élisabéthaines, comme peut en témoigner Pauvre Tom dans King Lear, ce premier type de représentation pose néanmoins trois problèmes de taille. Premièrement, une seule image (puisque seule la vignette de The Vow Breaker concerne directement le théâtre) est insuffisante pour émettre une quelconque hypothèse sur les conventions iconographiques et, à plus forte raison, dramatiques. Deuxièmement, le texte de The Vow Breaker contredit cette vignette, puisque Anne, empruntant ses mots à Shakespeare, précise bien à propos du spectre de Bateman qu'il apparaît comme de son vivant : "In the same figure that it used to be » (III.i.329). Troisièmement, un autre indice permet d'infirmer la suggestion de cet exemple unique: les inventaires de Philip Henslowe, patron du théâtre de la Rose, attestent l'existence d'un costume propre aux spectres. En effet, dans celui du 10 mars 1598, le gérant scrupuleux mentionne en rapport avec le fantôme un costume («gostes sewt [i.e. suit] »), un corset, ou sous-vêtement ample («gostes bodeyes [i.e. bodice]»), ainsi qu'une couronne («gostes

\footnotetext{
7 Lorsque ce même imprimeur fit publier, une douzaine d'années plus tard, une traduction du recueil de Lycosthènes, il réutilisa plusieurs gravures de Boaistuau, dont celle-ci (The Doome warning all men to the Iudgemente, Londres, Ralphe Nubery pour Henry Bynneman, 1581, p. 420).
} 
crown $»)^{8}$. Bien que très vagues, ces expressions suggèrent plutôt un costume générique, puisque pour signifier un spectre royal on a recours à une couronne spécifique.

\section{Suaires et draps : du tom beau à la chambre à coucher}

Paru peu avant le succès de Hamlet, un commentaire fort critique sur la figure spectrale laisse supposer l'existence de deux costumes distincts. Dans le préambule à une pièce anonyme, A Warning for Fair Women (1599), History, Tragedy et Comedy se disputent, et cette dernière brosse un portrait peu flatteur des activités de Tragedy qu'elle réduit à des histoires de tyrans et de meurtres :

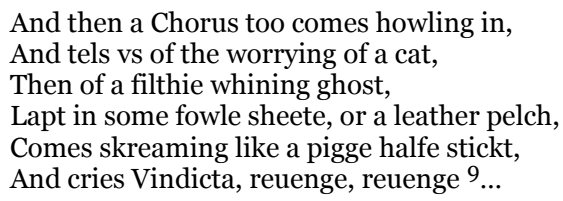

Si l'on identifie sans mal «fowle sheete» à un linceul souillé, l'alternative proposée pose problème, comme nous le verrons plus loin.

L'iconographie offre en effet une convention très fréquente pour représenter les revenants : le linceul. Dès le Moyen-Âge on trouve des enluminures qui représentent l'âme ainsi revêtue ${ }^{10}$. Sur la douzaine de gravures anglaises que j'ai pu recenser (la plupart assez tardives), on distingue clairement deux façons de porter le suaire. Le premier groupe l'arbore comme une toge, drapant le corps entier et recouvrant la tête. Il se limite en fait à une seule planche (Ill.7), mais reproduite ou imitée dans au moins cinq textes différents entre 1641 et $1655^{11}$. Le deuxième type la porte comme une grande pièce de tissu, nouée audessus de la tête, retombant sur le revenant et cachant en partie son

\footnotetext{
${ }^{8}$ Henslowe's Diary, éd. R. A. Foakes et R. T. Rickert, Cambridge, C.U.P., 1961, p. 319 et 321.

${ }^{9}$ A Warning for Fair Women, Londres, Valentine Sims pour William Aspley, 1599, Ind.4653.

${ }^{10}$ Voir note 4 ci-dessus.

${ }^{11}$ Il s'agit de deux ballades et de quatre pamphlets dont les personnages principaux sont des fantômes [T]he [de]puties ghost : or [his?] apparition to the [l]ord of Canterbury in the Tower (1641), Tom Nashe his Ghost (1642), The Ghost of Sr. John Presbiter (1647), Colonell Rainsborowes ghost (1648) et The Quakers Fiery Beacon: Or, the ShakingRanters Ghost (1655).
} 
corps. Ce type donne lieu à de nombreuses variations, soit sous forme de vignettes « recyclables » (IIl.8), soit dans des illustrations conçues plus ou moins explicitement pour les textes qu'elles accompagnent. Voici trois exemples allant vers le plus topique avec Strange Apparitions, or The Ghost of King James (1642) où les noms des différents personnages annoncés dans le titre sont inscrits également sur la gravure ${ }^{12}$ (Ill.9).

Pour ce qui est de la pratique théâtrale, l'étude systématique du corpus fait également ressortir le linceul comme le costume le plus répandu.

Ainsi, le premier à y faire allusion est Ben Jonson qui, en 1597 dans The Case is Altered, évoque dans une remarque ironique : " the ghost of some great Satrapas / In an vnsauory sheet. » (I.v.56-57). On peut rapprocher l'emploi de «vnsauory » de celui de «fowle » dans A Warning for Fair Wom en (1599). Très souvent, on observe au niveau des termes utilisés un glissement du linceul à la blancheur d'un drap, de « winding sheet » à « white sheet », au point que souvent c'est la blancheur et non plus le linge funéraire qui évoque le fantôme.

Je prendrai l'exemple de l'anonyme The Merry Devil of Edmonton, où un bedeau (Sexton), rentrant dans son église, aperçoit un meunier qui, épuisé, s'est réfugié sous le porche. Sans doute à cause de la blancheur de ce dernier (« a white thing »), il le prend pour un fantôme. Lorsque arrive le prêtre, le bedeau se confie à lui et le prêtre se moque de lui par la surenchère : «fifteen spirits in the forrest, like white bulles »(1130-1132). Dans la scène suivante, c'est au tour de l'aubergiste Blague de voir le meunier et sa réaction est la même, tant il est influencé par les croyances populaires :

What the deuill is that white thing ? this same is a Church-yard, and I haue heard that ghosts, and villenous goblins haue beene seene here ${ }^{13}$.

Là-dessus, le prêtre revient en compagnie du bedeau et, toujours sur un ton pince-sans-rire avance une hypothèse diabolique sur la nature du phénomène, soupçonnant : «the deuill, with a mans body vpon his

\footnotetext{
12 « The Ghost of King Iames, With a late conference between the ghost of that good King, the Marquesse Hameltons, and George Eglishams, Doctor of Physick, unto which appeared the ghost of the late Duke of Buckingham concerning the death and poysoning of King Iames and the rest » (Strange Apparitions, Londres, J. Ashton, 1642).

13 The Merry Devil of Edmonton, Londres, Henry Ballard pour Arthur Johnson, 1608, 1148-1150.
} 
backe in a white sheet » (1152-1153), et les allusions à la blancheur et aux spectres continuent.

La même scène se répète dans The Vow Breaker, lorsque Miles le meunier est pris pour un fantôme à cause de son accoutrement décrit comme un manteau de meunier farineux («mealy Millers coate», v.i.199-200).

Cette situation comique conforte le trait caractéristique de blancheur, mais ici l'ironie est redoublée par le personnage du meunier. Ses vêtements, blanchis par le métier qu'il exerce, jouent sur un autre code de représentation dans The Knight of the Burning Pestle (1607) de Francis Beaumont. Dans cette pièce, Jasper se fait passer pour son propre fantôme en appliquant de la farine sur son visage ${ }^{14}$. La farine peut donc servir à rendre blanc aussi bien un visage qu'un vêtement et, des années plus tard, ce procédé est évoqué de façon moins précise dans The Rebellion (1638) de Thomas Rawlins, où un personnage qui veut se faire passer pour un fantôme parle d'une petite ruse d'acteur : "A little Players deceite : flower will doe't15». S'il est juste d'y voir un commentaire des pratiques théâtrales, la méprise du bedeau et des autres personnages crédules permet en même temps de tourner en dérision le moyen même de représenter un spectre sur scène.

Le linceul blanc peut donc se confondre aisément avec la blancheur générale du revenant, d'autant que certains dramaturges exploitent des apparitions de spectres féminins, qui font écho aux apparitions des premiers martyrs chrétiens, voire à celle de la Vierge immaculée, comme dans l'anonyme The Second Maiden's Tragedy, The Virgin Martyr de Massinger ou encore Messalina de Richards. Au fil des années le véritable linceul, les vêtements recouverts de farine ou un visage très pâle (probablement maquillé à l'aide de la même farine) deviennent des techniques équivalentes pour signifier la blancheur du fantôme, celle-ci devenant le trait caractéristique du costume.

L'utilisation des costumes pour représenter un spectre, et en particulier du linceul, trouve un précieux éclairage dans les pièces où des personnages se déguisent en fantômes ou bien évoquent un tel

\footnotetext{
14 «Enter Jasper and his face mealed» (The Knight of the Burning Pestle, éd. Michael Hattaway, Londres, A \& C Black, coll. « New Mermaids », 1986, v.i.4).

15 The Rebellion, Londres, I. Okes pour Daniell Frere, 1640, v.i.107-108.
} 
déguisement sans le mettre en pratique. Un tel contexte permet de plus un jeu polysémique entre le drap mortuaire du tombeau et le drap blanc de la chambre à coucher.

Dans The Atheist's Tragedy, soucieux de ne pas être dérangé dans ses ébats amoureux avec Soquette, Languebeau Snuffe apporte avec lui quelques attributs dont un drap, une perruque, et une barbe («pulls out a sheet, a hair, and a beard» IV.iii.56 $6^{\mathrm{D} 16}$ ) pour faire peur aux indésirables, en se faisant passer pour le spectre de Montferrers. D’ailleurs, pour satisfaire la curiosité de sa bien-aimée, il les revêt surle-champ, ce qui fournit à Tourneur l'occasion de parodier Hamlet dans la réaction de la jeune fille :

Snu. How do I looke in this habit, wench?

Soq. So like a Ghost that, notwithstanding I haue som foreknowledge of you, you make my hair stand almost on end. (IV.iii.63-66)

De même, lorsque Tom Lurcher et Boy discutent du déguisement à mettre pour le cambriolage dans The Night Walker (Le Rôdeur Nocturne) (1611) de John Fletcher, ce dernier propose entre autres «A winding sheet " (II.56). Par la suite, le lien avec le cadavre semble devenir plus explicite, puisque ceux qui se font passer pour des revenants se cachent en plus dans des cercueils, comme c'est le cas dans Love's Sacrifice (1632) de John Ford ou dans l'anonyme The Ghost (1640).

Le linceul comme emblème du déguisement en fantôme, plus que d'un «véritable » revenant, est un motif que l'on retrouve aussi chez les démonologues. Ils associent systématiquement l'utilisation du linceul à la tromperie quand des personnes mal intentionnées se font passer pour des spectres. Lavater évoque cette pratique dès 1569 :

Many times, pleasant \& merrie yong men, disguise themselues like vnto Deuils, or else shroud themselues in white sheetes to make other men afrayde: with whome if simple men chaunce to meete, they make no doubt of the matter, but verily thinke they haue seene spirites, and straunge sightes ${ }^{17}$.

\footnotetext{
${ }^{16}$ In Four Revenge Tragedies, éd. Katharine Eisaman Maus, Oxford, O.U.P., 1995.

${ }^{17}$ Lavater, Trois livres des apparitions, Zurich, Guillaume des Marescz, 1581, liv. I, chap. iv, p. 16.
} 
Reginald Scot, pour sa part, parle de «some one knave in a white sheete hath cousened and abused manie thousands that waie ${ }^{18}$ ».

Les rares références littéraires à une telle apparence - en dehors du théâtre - sont faites également dans un contexte de fauxsemblant ${ }^{19}$. Dans des ballades populaires, par exemple, on peut trouver le détournement du linceul à des fins amoureuses ${ }^{20}$. Rien d'étonnant dès lors à ce que le suaire apparaisse comme un procédé propre au théâtre: le déguisement sous toutes ses formes y tient un rôle fondamental, et l'utilisation de cet accessoire dans la représentation d'un fantôme permet indirectement de rappeler qu'il s'agit avant tout d'un acteur qui joue un rôle.

\section{The White Devil : exception ou parodie?}

Ayant commencé l'examen du costume spectral par une exception notoire, on ne pouvait le terminer que par une autre exception, un peu moins mémorable. En dehors de Ham let, une seule autre pièce, The White Devil de John Webster, prend la peine de préciser la tenue d'un fantôme, et la démarche est très différente de celle de Shakespeare. Lorsque le spectre de Brachiano entre sur scène, une didascalie spécifie très clairement son apparence :

Enter Bracciano's Ghost, in his leather cassock and breeches, boots, [and] a cowl [in his hand] a pot of lilly-flowers with a skull in't. $(\text { V.iv.119 })^{21}$

Cet accoutrement diffère très clairement de ce que Brachiano portait de son vivant. Dans la scène précédente, on le voit d'abord en armure, puis on le revoit sur son lit de mort, ce qui, à première vue, rend peu probable la tenue décrite dans la didascalie.

Le capuchon $(\operatorname{cow} l)$ peut être perçu comme un écho visuel de la scène précédente dans laquelle Lodovico et Gasparo, déguisés en

\footnotetext{
18 The Discoverie of Witchcraft, Londres, William Brome, 1584, liv. vII, chap. xv, p. 86.

${ }^{19}$ Dans The Wonderful Year (1603), Thomas Dekker parle de " Ghosts in white sheetes of paper» (p. 5) et dans Scoggin's Jests (1613), il décrit un personnage qui se déguise pareillement pour extorquer de l'argent à une veuve : " he came into her house \& lapped himselfe in a white sheete, counterfetting a spirit » (p. 38).

${ }^{20}$ Willie's Lyke-Wake (25) in Child, The English \& Scottish Popular Ballads, Boston, Houghton, Mifflin \& Co, 1882-1898, vol. I, p. 250-251.

${ }^{21}$ In John Webster, The Duchess of Malfi and other Plays, éd. René Weis, Oxford, O.U.P., coll. « Oxford World's Classics », 1996.
} 
moines capucins ( « in the habit of Capuchins »), étranglent Brachiano. Il y a de la part de Webster un souci évident de rapprocher ces deux scènes puisqu'une remarque de Flamineo dans la scène précédente préfigurait déjà l'apparition à venir ${ }^{22}$. Le capuchon peut alors apparaître comme une tentative de la part de Brachiano de dénoncer ses meurtriers, d'autant plus que contrairement à hood, cowl ne s'emploie à l'époque que pour désigner un vêtement religieux, systématiquement accolé à friar ou monk .

Jean MacIntyre propose une lecture fondamentalement différente de ce capuchon qu'elle préfère associer aux autres habits portés par le spectre, l'ensemble constituant selon elle la tenue militaire de Brachiano une fois l'armure retirée, ce qui a de plus l'avantage de faciliter le changement de costume entre les deux scènes ${ }^{23}$. Cowl et cassock deviennent ainsi des vêtements portés sous l'armure au même titre que breeches. Si l'hypothèse parait séduisante et très fonctionnelle, elle soulève néanmoins un problème d'usage. D'une part, cowl apparaît essentiellement dans le contexte religieux et non guerrier; de l'autre, cassock désigne une grande variété de vêtements, dont nous retiendrons deux acceptions principales. Dans le contexte militaire un manteau qui, au contraire, recouvre l'armure ; dans le contexte religieux un sous-vêtement ample que l'on met sous la soutane ${ }^{24}$, comme dans The Devil's Charter (1607) de Barnabe Barnes où une didascalie montre le pape Alexandre justement dans cet habit: "Alexander vpon the stage in his cassock and nightcap " (IV.v.74 ${ }^{\mathrm{D}}$ ). On se rappellera un autre élément de l'inventaire de Henslowe qui semble pouvoir recouvrir ce type de vêtement - « ghoste bodeyes »c'est à dire un sous-vêtement ressemblant à un corset. Le problème reste que Webster indique un vêtement en cuir, ce qui ne correspond ni aux étoffes précieuses du manteau militaire, ni à une bure de moine.

Dans sa définition de casaca, Sebastián de Covarrubias glose le terme par le latin pellis. Or, cette racine latine, ainsi que l'expression « leather cassock» ne manquent pas d'évoquer un autre manteau en

\footnotetext{
22 Voir v.iii.125-127.

23 Costumes and Scripts in the Elizabethan Theatres, Edmonton, University of Alberta Press, 1992, p. 267-268.

24 Bien que l'OED situe la première occurrence de cette acception en 1663, la définition qu'en donne le dictionnaire français-anglais de Cotgrave, publié en 1611, ne laisse aucun doute: «Soutane: [f.] A long and loosse coat, or cassocke, such as Church-men weare vnder their gownes. "
} 
cuir que l'auteur anonyme de A Warning for Fair Women voyait comme l'apanage des fantômes de théâtre : " a leather pelch ». Il est peu probable qu'il s'agisse d'une coïncidence, mais on ne peut pas en conclure pour autant, comme le fait René Weis dans son édition des pièces de Webster, que c'était l'habit typique des spectres sur scène ${ }^{25}$. En effet, A Warning for Fair Women précède The White Devil de plus d'une dizaine d'années, et il n'existe aucune autre pièce qui mentionne cet élément de garde-robe fantomatique ${ }^{26}$. Il est plus logique de penser que Webster s'inspire de A Warning for Fair Women pour représenter un revenant caricatural, plus proche des descriptions qu'on en fait que des pratiques théâtrales ${ }^{27}$. Que Webster ait eu ici des intentions ironiques est fortement suggéré par la réaction de Flamineo : «What a mockery hath death made of thee ? » (v.iv.121).

Finalement, puisque les occurrences de «leather pelch» relèvent du contexte parodique, ce vêtement, que l'on imagine sali et jaunâtre, est une sorte de pendant au linceul blanc souillé par l'inhumation et par le temps, les deux costumes offrant des caractéristiques communes. Jean MacIntyre parvient aux mêmes conclusions à partir de quelques mises en scène en soulignant qu'il devait nécessairement s'agir d'un costume très simple à utiliser, car certains personnages disposent de très peu de temps pour se changer (surtout dans le cas du doublage de rôles) ${ }^{28}$. Le suaire et le manteau remplissent ces conditions parce qu'on peut les enfiler par-dessus un autre costume.

Tous ces éléments, parfois contrastés, aboutissent à un tableau assez cohérent et homogène si l'on excepte le cas Hamlet. Le linceul est généralement de rigueur, cédant parfois la place à un vêtement plus imprécis, mais qui en conserve les principaux traits. Cette « casaque »

25 Cf. «leather cassock : a long loose coat worn by ghosts » (op. cit., p. 385). Il n'est d'ailleurs pas le seul. Dans leur édition de Webster, Jonathan Dollimore et Alan Sinfield faisaient déjà le même constat hâtif : «leather cassock: customary dress for a ghost » (Selected Plays, Cambridge, C.U.P., 1983, p. 118).

26 Jean MacIntyre rappelle l'existence dans les inventaires de Henslowe de « j gostes sewt, and $\mathrm{j}$ gostes bodeyes ", mais précise également qu'il n'y a aucun moyen de savoir à quoi ces articles correspondaient (op. cit., p. 22 et Henslowe's Diary, op. cit., p. 318).

27 C'est le sentiment de Robert William Dent : « Webster evidently intended a grotesque, though perhaps common, juxtaposition of symbols » (John Webster's Borrowing, 1960, p. 161).

28 MacIntyre, op. cit., p. 22. 
est ample, facile à enfiler par-dessus un autre costume, produisant un effet semblable au drapé d'un suaire, qu'elle désigne un sous-vêtement en lin ou coton, ou bien un survêtement en cuir. Ce costume est complété par un maquillage à base de farine visant à rendre la pâleur du défunt, l'utilisation de la farine pouvant aussi s'étendre aux vêtements et autres éléments de la garde-robe spectrale, souvent rehaussée par l'ajout du sang. Ainsi, la couronne de fantôme que Henslowe mentionne dans ses inventaires pourrait bien être « blanchie » pour cet usage, ce qui suffirait à la rendre différente des autres couronnes. Avec le succès de la figure spectrale à partir des années 1580 , ce costume se généralise en accentuant le contraste avec l'accoutrement des esprits malins qui ont systématiquement recours au noir et au masque. En somme, le théâtre anglais développe des codes de représentation qui puisent dans les croyances ancestrales, aussi bien païennes que catholiques, sans tenir compte des théories protestantes en vogue qui rejettent le retour des âmes, assimilant toute apparition à l'œuvre du Malin. Quant à Hamlet, par son succès au-delà de la Renaissance, la pièce a aussi un effet déformant sur notre perception de la figure spectrale, car elle fait souvent oublier la réalité d'une pratique dont elle s'écarte. Si Hamlet enrichit ce motif dramatique, il ne le modifie pas radicalement.

Pierre KAPITANIAK Université Paris VIII 